\title{
Overexpression of Human DNA Topoisomerase II $\alpha$ by Fusion to Enhanced Green Fluorescent Protein
}

BioTechniques 25:1052-1057 (December 1998)

\author{
Yin-Yuan Mo, Keith A. \\ Ameiss and William T. Beck \\ University of Illinois at \\ Chicago, Chicago, IL, USA
}

\section{INTRODUCTION}

DNA topoisomerase (topo) II is an essential enzyme that regulates DNA topology for DNA replication and transcription during cell growth and cell cycle progression (8). In mammalian cells, although two types of topo II exist, $\alpha$ and $\beta(5,10)$, topo II $\alpha$ seems to be the predominant isoenzyme in most tissues. Its clinical importance comes from findings that this enzyme is a major target for many anticancer drugs (7). Although much information about the physiological and biochemical aspects of the enzyme has been accumulated from studies of the yeast and Drosophila enzyme, little is known about regulation and expression of the gene for human topo II $\alpha$ in mammalian systems. This unfamiliarity has been attributed to the difficulty in expressing the topo II $\alpha$ gene in mammalian systems. For example, several mutations in human topo II $\alpha$ have been identified in drugresistant cell lines (9), but testing whether any of these mutations confer drug resistance has not been possible in mammalian systems.

Human topo II $\alpha$ is $170-\mathrm{kDa}$ nuclear protein consisting of 1530 amino acids (10). Although some success has been reported in yeast systems (4), as with many other large proteins, expression of this gene in mammalian cells has been a great challenge. While the expression of human topo II $\alpha$ in mammalian cells has been reported (1), the results were difficult to interpret because the exogenous gene product was indistinguishable from the endogenous counterpart. To express human topo II $\alpha$ in human cells, we have tried a variety of approaches, including use of constitutive strong, weak or inducible promoters (e.g., cytomegalovirus [CMV], Rous sarcoma virus [RSV] and tetracycline, respectively) without success. All these efforts only led to expression of this gene at the transcriptional level, and no exogenous topo II $\alpha$ was detected by Western blot using antibody specific to the exogenous protein (Y.-Y. Mo and W.T. Beck, unpublished results). However, while we were examining expression of the C-terminal region of topo II $\alpha$ for its subcellular localization, we fused the $\mathrm{C}$ terminus of the gene to the enhanced green fluorescent protein (EGFP) and found very high expression of the fusion protein. We subsequently examined the possibility of expressing the full-length human topo II $\alpha$. By using the EGFP fusion, we were able to detect high levels of exogenous topo II expression at both the mRNA and protein levels in mammalian cells. This protein localized to the cell nucleus and appeared to be catalytically active, because it conferred sensitivity to the specific inhibitor of topoisomerase II, teniposide (VM-26).

\section{MATERIALS AND METHODS}

\section{Construction of Expression Plasmids}

To label human topo II $\alpha$, we introduced the RGS- $6 \times$ His sequence from a QIAexpress ${ }^{\mathrm{TM}} \mathrm{pQE}$ high level bacterial 
expression vector (Qiagen, Chatsworth, CA, USA) at KpnI and BamHI sites of pCDNA3 (Invitrogen, Carlsbad, CA, USA) to make an N-terminal tag (pCDNA3-His). This was done so that this fragment could be released by digestion with these two enzymes. For efficient translation of the protein, the Kozak sequence (6) was introduced in the front of the RGS- $6 \times$ His tag (Figure 1). To facilitate the subcloning of topo II $\alpha$ cDNA into pCDNA3-His, a BamHI site was introduced at the $\mathrm{N}$ terminus of topo II $\alpha$ cDNA by polymerase chain reaction (PCR) amplification, eliminating the first methionine (start codon) of topo II $\alpha$. Similarly, the restriction sites SacII and NotI were introduced at the C terminus after the stop codon. Primers for the $\mathrm{N}$-terminal sequence were: IIa-B1 (sense) 5'-GGATCCGAAGTGTCACCATTGCAG-3' and IIa-3.1 (antisense) 5'-GGTGGATCCAGCAATATCAT-3'; primers for the C terminus were IIa-C1A (sense) 5'-GTGACAGTGAAGAAGACAGCAG-3' and IIa-C18.1 (antisense) 5'-GCGGCCGCGGTTAAAACAGATCATCTTCATC-3'. Sequences of the PCR products were verified by DNA sequencing.
Plasmid pT14 was constructed by cloning the full-length topo II $\alpha$ cDNA (10) in pCDNA3-His unidirectionally at BamHI and NotI sites. To construct pT104, pT14 was completely digested with SacII but partially digested with $H i n d I I I$, and the full-length topo II $\alpha$ cDNA plus RGS- $6 \times$ His was fused inframe to pEGFP-C3 (CLONTECH Laboratories, Palo Alto, CA, USA) at HindIII and SacII sites. Although this construct contains an internal start codon (ATG), the internal translational product was not detectable.

\section{Transfection, RT-PCR, Immunoblotting and Fluorescent Microscopy}

Expression plasmids were introduced into HeLa cells by the calcium phosphate method using $20 \mu \mathrm{g}$ DNA per culture dish (100 $\mathrm{mm}$ in diameter). Transfection efficiency was monitored by co-transfection of pT14 with pEGFP-C3 or by pT104 alone. Cells were harvested $16 \mathrm{~h}$ posttransfection for either RNA isolation or protein extraction. Total RNA was isolated by using TRIzol ${ }^{\circledR}$ Reagent (Life Technolo- gies, Gaithersburg, MD, USA) per the manufacturer's protocol. Contaminating DNA in the RNA samples was removed by treatment with RNase-free DNase before reverse transcription (RT). Primers used to detect exogenous topo II $\alpha$ by RT-PCR were: T1 (sense), 5'-GGTACCGATACCATGCGGGGTTCT-3' (Figure 1), derived from the vector sequence and $\mathrm{T} 2$ (antisense) $5^{\prime}$ TAGCCATTTCGACCACCTGTCAC$3^{\prime}$, derived from the topo II $\alpha$ sequences. This set of primers allows us to amplify only the exogenous gene (ca. $500 \mathrm{bp}$ ) at the $\mathrm{N}$ terminus. For Western blotting, total proteins were extracted by lysing cells in dishes with $1 \times$ sample buffer $(50 \mathrm{mM}$ Tris- $\mathrm{HCl}, \mathrm{pH}$ $6.8,2 \%$ sodium dodecyl sulfate [SDS], and $10 \%$ glycerol), and the protein concentration was measured by a Protein Assay Kit (Bio-Rad, Hercules, CA, USA). Approximately $50 \mu \mathrm{g}$ of proteins were loaded in each lane of a $7 \%$ SDS polyacrylamide gel. To detect exogenous proteins, we used anti-RGSHis antibody (Qiagen) and GFP antibody (CLONTECH), respectively; antibody 284 (2) was used to detect both endogenous and exogenous topo II $\alpha$.

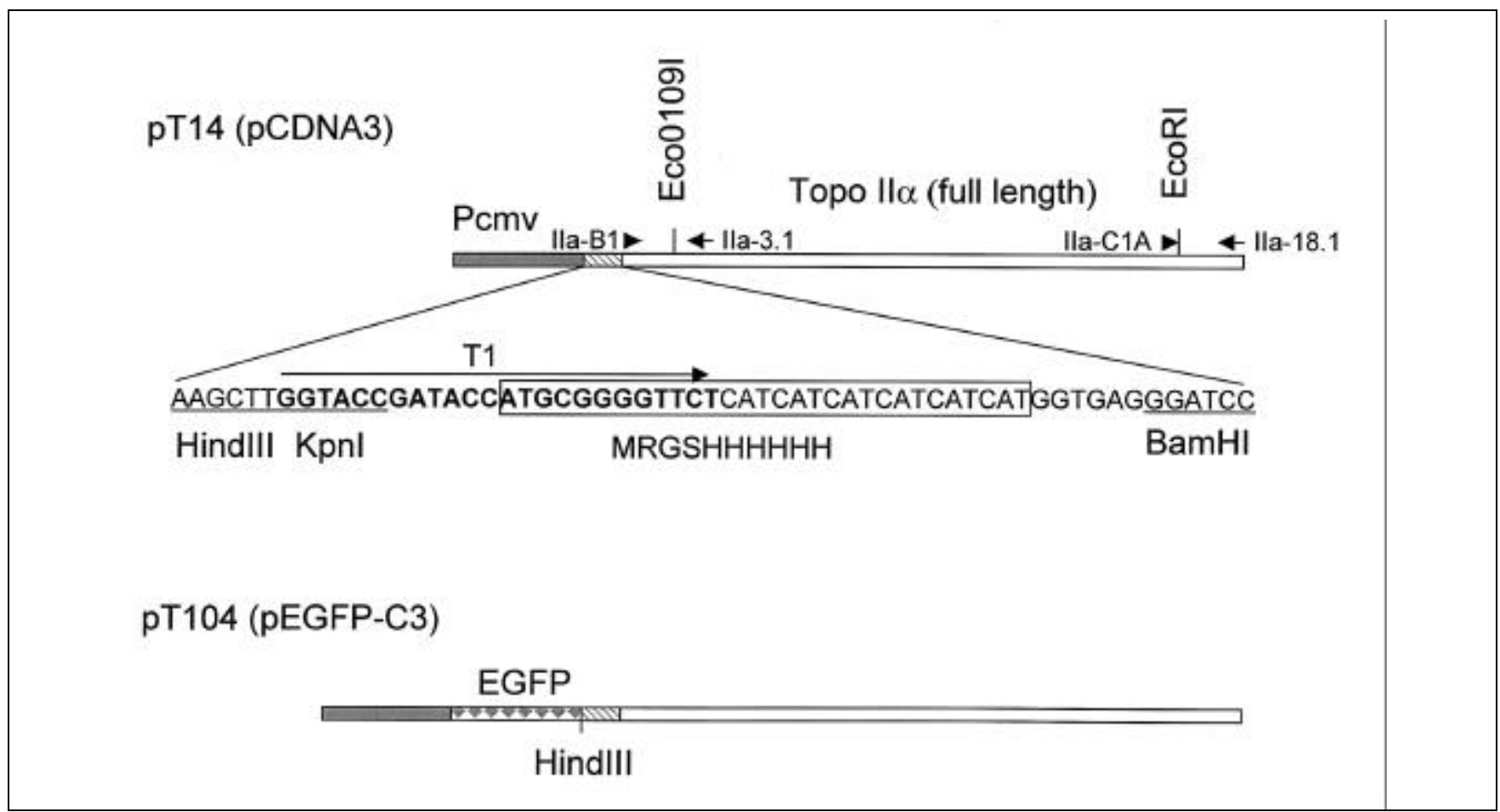

Figure 1. Constructs of human topo II $\alpha$ used in transfection experiments. Primers used for amplifying the N- or C-terminal regions of topo II $\alpha$ by RT-PCR are shown as arrows. Restriction sites (Eco0109I and EcoRI) were used for convenient cloning of PCR products. RGS-6×His sequence is blocked. As indicated, both constructs use the same CMV promoter (Pcmv), but pT104 carries EGFP fused to topo II $\alpha$. The drawing is not in scale. 
Signals were visualized with ECL ${ }^{\mathrm{TM}}$ Western Blotting Detection Reagents (Amersham Pharmacia Biotech, Piscataway, NJ, USA). Subcellular localization of the fusion protein in living cells was monitored under an Axioskop ${ }^{\circledR}$ fluorescent microscope (Carl Zeiss, Thornwood, NY, USA) using a filter with maximum excitation at $480 \mathrm{~nm}$ and maximum emission at $520 \mathrm{~nm}$.

\section{Growth Inhibition Assay}

HeLa cells were transiently transfected with topo II $\alpha$ constructs and seeded into 12-well plates at approximately 0.2 million cells per well. After $24 \mathrm{~h}$ of treatment with VM-26 at various concentrations, cells were trypsinized, and cell numbers were determined by using the Coulter ${ }^{\circledR}$ Counter (Beckman-Coulter, Miami, FL, USA).

\section{RESULTS AND DISCUSSION}

Because it is a large protein, topo II $\alpha$ is difficult to express exogenously. A general consensus view is that expression of exogenous topo II $\alpha$ is cytotoxic and that it is strictly regulated through the cell cycle (3). However, transient expression is presumably not affected by these factors. Therefore, we sought to express topo II $\alpha$ in transiently transfected HeLa cells. To distinguish exogenous topo II $\alpha$ from its endogenous counterpart, we tagged topo II $\alpha$ with RGS- $6 \times$ His at the $\mathrm{N}$ terminus (pT14; see Figure 1); an antibody against this tag is commercially available. When pT14 was introduced into HeLa cells, we consistently detected exogenous topo II $\alpha$ mRNA by RT-PCR using a set of primers that specifically amplify the $6 \times$ His-tagged topo II $\alpha$. Figure $2 \mathrm{~A}$ shows an example of such RT-PCRs. This approximately 500-bp fragment specifically came from expression of the exogenous topo II $\alpha$ since no product was seen in PCR using the same RNA sample without RT. Similarly, we saw no products with RNA isolated from cells transfected with vector alone (data not shown). However, no exogenous topo II $\alpha$ protein was detected by antiHis antibody (Figure 2B). Even for some deletion constructs, we detected no expression of exogenous topo II $\alpha$ protein (data not shown).

By contrast, when the same fragments were fused to EGFP, we found that they were expressed well (data not shown), indicating that EGFP appears to enhance expression of the exogenous gene at the protein level. Consequently, we cloned the full-length topo II $\alpha$ cDNA into pEGFP-C3 (pT104; see Figure 1). Figure $2 \mathrm{~A}$ shows that, as with pT14, RT-PCR detected comparable exogenous topo II $\alpha$ mRNA from pT104-transfected cells. Figure 2B

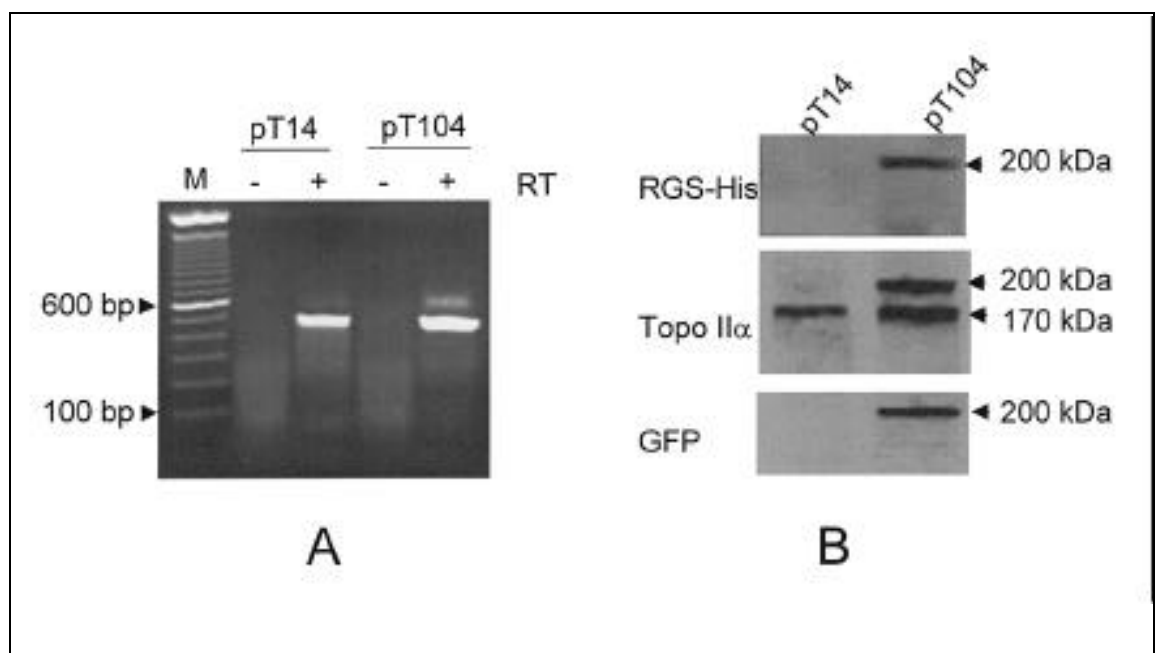

Figure 2. Transient expression of topo II $\alpha$ in HeLa cells. (A) Expression of exogenous topo II mRNA as detected by RT-PCR. An equivalent of $10 \mathrm{ng}$ of total RNA were used for RT-PCR to generate the products seen in each lane. RT, reverse transcription; M, 100-bp DNA markers. (B) Detection of expressed exogenous topo II $\alpha$ protein by Western blot using antibodies specific to topo II $\alpha$, RGS- $6 \times$ His or GFP as indicated on the left.

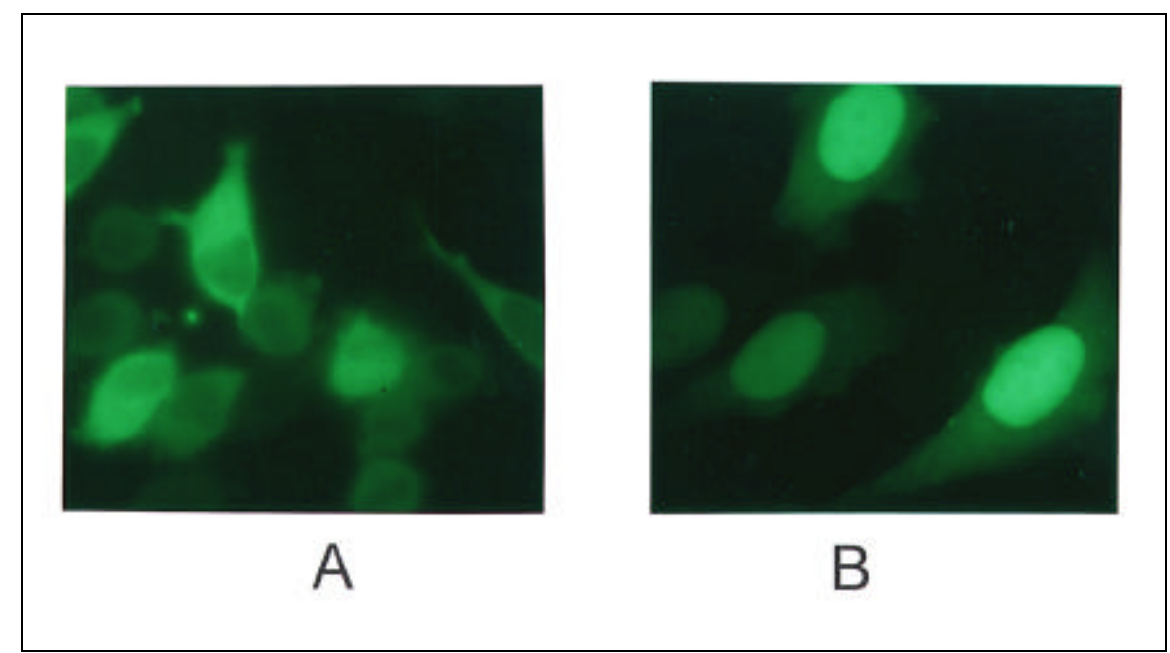

Figure 3. Nuclear localization of EGFP-topo II $\alpha$ fusion protein by fluorescent microscopy. HeLa cells were grown overnight on cover slips in culture dishes, followed by transfection with either pT14 plus pEGFP-C3 (A) or pT104 alone (B). Sixteen hours after transfection, cover slips were transferred to glass slides, and expression of EGFP was monitored by fluorescent microscopy. Notice nuclear localization of the EGFP-topo II $\alpha$ fusion protein in Panel B. 


\section{Cancer Research Techniques}

were detected by this antibody in pT104-transfected cells (Figure 2B, middle panel). To further verify that the $200-\mathrm{kDa}$ protein is the EGFP-topo II $\alpha$ fusion protein, an anti-GFP antibody was used to probe the membrane that was stripped for a second time. As with the anti-RGS- $6 \times$ His antibody, the antibody against GFP reacted with only the $200-\mathrm{kDa}$ band but not the $170-\mathrm{kDa}$ band (Figure 2), suggesting that the $200-\mathrm{kDa}$ band is the exogenous EGFPtopo II $\alpha$ and that the signals from the previous blot were completely removed.

To determine whether low transcription activity leads to undetectable expression of pT14, we used the same set of primers that only amplify the exogenous topo II $\alpha$ cDNA. Semiquantitative
RT-PCR suggested that about same amount of exogenous topo II $\alpha$ mRNA was made for both pT14 and pT104 (data not shown). To exclude the possibility that lack of expression of pT14 could be due to a low transfection efficiency, we determined transfection efficiency by co-transfecting pT14 with pEGFP-C3, because pT14 does not have a monitoring marker. By contrast, the pT104 has the EGFP marker to use to determine transfection efficiency with this construct alone. Average transfection efficiency calculated from more than 10 independent experiments was about $25 \%$. In fact, the efficiency for pT14 was slightly higher than that for pT104. Figure 3 shows expression of these markers following transfection into HeLa cells. As expected, the EGFP-topo II $\alpha$ fusion protein was localized to the cell nucleus, suggesting that the protein is folded properly. Finally, we tested the functionality of the EGFP-topo II $\alpha$ protein by growth inhibition assay. Overexpression of the EGFP-topo II $\alpha$ increases the cell's sensitivity to the topo II inhibitor VM-26 (Figure 4). Inhibitory concentration (IC) $_{50}$ for the control was $4.6 \mu \mathrm{M}$, whereas $\mathrm{IC}_{50}$ for pT104-transfected cells was $0.9 \mu \mathrm{M}$, suggesting that this fusion protein functions as the endoge-

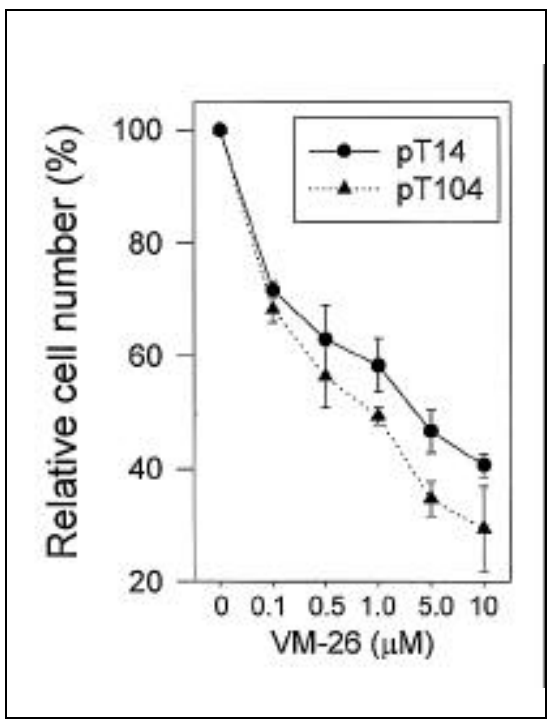

Figure 4. Effect of expression of the topo IIo on sensitivity of cells to VM-26. HeLa cells were transfected with pT14 or pT104 and then treated with VM-26 at the indicated concentrations for $24 \mathrm{~h}$. Data are the means $( \pm$ standard deviation) of three independent experiments. 
nous counterpart. Therefore, we demonstrated here that GFP is a valuable tool not only for gene tagging, but also for enhancing the expression of such difficult genes as topo II $\alpha$.

Achieving overexpression of some genes is often challenging and thus hinders studies of the gene products' physiological functions. Our results suggest that some of these genes might be able to be expressed using this GFP fusion approach. Indeed, our preliminary results with overexpression of topo II $\beta$ using the same approach seem to support this notion (Y.-Y. Mo and W.T. Beck, unpublished data). The reason for GFP fusion enhancement of topo II $\alpha$ expression is not clear at this point. Since topo II $\alpha$ mRNA levels for both constructs are about the same, it is possible that EGFP either increases translation efficiency or facilitates protein folding, thus stabilizing the protein and preventing degradation. Because both constructs carry the same Kozak sequence in front of the translation start codon (ATG), they should be translated at the same efficiency. If this is the case, EGFP might stabilize the overexpressed topo II $\alpha$ gene product.

\section{ACKNOWLEGDMENTS}

This work was supported in part by Research Grant No. ACS97-34 from the American Cancer Society, Illinois Division (Chicago, IL, USA) to Y-Y. Mo, and by Research Grant No. CA40570 from the National Cancer Institute, NIH, Department of Health and Human Services (Bethesda, MD, USA) to W.T. Beck, and in part by the Cancer Center, University of Illinois at Chicago. We thank Ena Yoon for her technical assistance.

\section{REFERENCES}

1.Asano, T., T. An, J. Mayes, L.A. Zwelling and E.S. Kleinerman. 1996. Transfection of human topoisomerase II $\alpha$ into etoposide-resistant cells: transient increase in sensitivity followed by down-regulation of the endogenous gene. Biochem. J. 319:307-313.

2.Boege, F., A. Andersen, S. Jensen, R. Zeidler and H. Kreipe. 1995. Proliferation-associated nuclear antigen $\mathrm{Ki}-\mathrm{S} 1$ is identical with topoisomerase II $\alpha$ : delination of a carboxylterminal epitope with peptide antibodies. Am.
J. Pathol. 146:1302-1308.

3.Goswami, P.C., J.L. Roti and C.R. Hunt. 1996. The cell cycle-coupled expression of topoisomerase II alpha during $\mathrm{S}$ phase is regulated by mRNA stability and is disrupted by heat shock or ionizing radiation. Mol. Cell. Biol. 16:1500-1508.

4.Hsiung, Y., M. Jannatipour, A. Rose, J. McMahon, D. Duncan and J.L. Nitiss. 1996. Functional expression of human topoisomerase II alpha in yeast: mutations at amino acids 450 or 803 of topoisomerase II alpha result in enzymes that can confer resistance to anti-topoisomerase II agents. Cancer Res. 56:91-99.

5.Jenkins, J.R., P. Ayton, T. Jones, S.L. Davies, D.L. Simmons, A.L. Harris, D. Sheer and I.D. Hickson. 1992. Isolation of cDNA clones encoding the beta isozyme of human DNA topoisomerase II and localization of the gene to chromosome 3 p24. Nucleic Acids Res. 20:5587-5592.

6.Kozak, M. 1987. At least six nucleotides preceding the AUG initiator codon enhance translation in mammalian cells. J. Mol. Biol. 196:947-950.

7.Liu, L.F. 1989. DNA topoisomerase poisons as antitumor drugs. Annu. Rev. Biochem. 58:351-375.

8.Nelson, W.G., L.F. Liu and D.S. Coffey. 1986. Newly replicated DNA is associated with DNA topoisomerase II in cultured rat prostatic adenocarcinoma cells. Nature 322:187-189.

9.Nitiss, J.L. and W.T. Beck. 1996. Antitopoisomerase drug action and resistance. Eur. J. Cancer 6:958-966.

10.Tsai-Pflugfelder, M., L.F. Liu, A.A. Liu, K.M. Tewey, J. Whang-Peng, T. Knutsen, K. Huebner, C.M. Croce and J.C. Wang. 1988. Cloning and sequencing of cDNA encoding human DNA topoisomerase II and localization of the gene to chromosome region 17q21-22. Proc. Natl. Acad. Sci. USA 85:7177-7181.

Received 23 June 1998; accepted 10 September 1998.

\section{Address correspondence to:}

Dr. William T. Beck

Division of Developmental Therapeutics

Cancer Center (M/C 569)

University of Illinois at Chicago

Chicago, IL 60607, USA

Internet: wtbeck@uic.edu 